This item was submitted to Loughborough's Research Repository by the author.

Items in Figshare are protected by copyright, with all rights reserved, unless otherwise indicated.

\title{
Dynamical density functional theory for the dewetting of evaporating thin films of nanoparticle suspensions exhibiting pattern formation
}

\section{PLEASE CITE THE PUBLISHED VERSION}

http://dx.doi.org/10.1103/PhysRevE.81.021602

\section{PUBLISHER}

(C) The American Physical Society

\section{VERSION}

VoR (Version of Record)

\section{PUBLISHER STATEMENT}

This work is made available according to the conditions of the Creative Commons Attribution-NonCommercialNoDerivatives 4.0 International (CC BY-NC-ND 4.0) licence. Full details of this licence are available at: https://creativecommons.org/licenses/by-nc-nd/4.0/

\section{LICENCE}

CC BY-NC-ND 4.0

\section{REPOSITORY RECORD}

Archer, Andrew J., Mark J. Robbins, and Uwe Thiele. 2019. "Dynamical Density Functional Theory for the Dewetting of Evaporating Thin Films of Nanoparticle Suspensions Exhibiting Pattern Formation". figshare. https://hdl.handle.net/2134/15988. 


\title{
Dynamical density functional theory for the dewetting of evaporating thin films of nanoparticle suspensions exhibiting pattern formation
}

\author{
A. J. Archer, M. J. Robbins, and U. Thiele \\ Department of Mathematical Sciences, Loughborough University, Leicestershire LE11 3TU, United Kingdom \\ (Received 22 May 2009; revised manuscript received 16 September 2009; published 4 February 2010)
}

\begin{abstract}
Recent experiments have shown that the striking structure formation in dewetting films of evaporating colloidal nanoparticle suspensions occurs in an ultrathin "postcursor" layer that is left behind by a mesoscopic dewetting front. Various phase change and transport processes occur in the postcursor layer that may lead to nanoparticle deposits in the form of labyrinthine, network, or strongly branched "finger" structures. We develop a versatile dynamical density functional theory to model this system which captures all these structures and may be employed to investigate the influence of evaporation or condensation, nanoparticle transport, and solute transport in a differentiated way. We highlight, in particular, the influence of the subtle interplay of decomposition in the layer and contact line motion on the observed particle-induced transverse instability of the dewetting front.
\end{abstract}

DOI: 10.1103/PhysRevE.81.021602

PACS number(s): 68.08.Bc, 05.20.Jj, 47.20.Hw, 47.57.-s

How surface patterns and structures evolve over time is of great interest for a wide range of scientific fields. Striking examples include river network patterns [1], the growth of rocks around geothermal springs [2], evaporation-caused coffee stain patterns [3], and the patterns in the distribution of living organisms [4]. Many such structures are generated by the interaction of fluid motion over the surface and deposition and/or abrasion of material. A particular process of high recent interest that concerns us in this paper is the formation of structures during the (evaporative) dewetting of nanoparticle suspensions on solid substrates [5-7]. The patterning is generic to a wide class of dewetting evaporating suspensions and solutions [8-12] and depends crucially on the interplay of several competing phase-change and transport processes.

The rapidly expanding study of such systems currently receives strong impetus from research in two distinct areas. On the one hand, studies from the last couple of decades of the dynamics of dewetting of surfaces by nonvolatile liquids $[13,14]$ have been extended to investigate the interplay between (de)wetting and evaporation of volatile liquids $[15,16]$. On the other hand, there is interest in the nonequilibrium thermodynamics and rheology of the respective phase and flow behavior of bulk suspensions and solutionssee, e.g., Ref. [17] and references therein. A thin film of pure nonvolatile liquid that is deposited upon a smooth substrate (e.g., a polystyrene film having a thickness of a few tens of nanometers, deposited on silicon oxide [13]) may rupture due to effective molecular interactions between the film surface and the solid substrate. The rupture mechanism can be (i) via a surface instability (often called spinodal dewetting) that occurs spontaneously and results in patterns of a certain characteristic wavelength or (ii) via nucleation at randomly distributed defects $[18,19]$. The resulting holes then grow to form a polygonal network of liquid rims that may subsequently decay into drops. All stages of this process are intensively studied: the rupture mechanisms $[13,14,18]$, the hole growth [20], the morphologies and evolution of the resulting patterns [21], and the stability of receding liquid fronts $[22,23]$. For reviews of this body of work, see Ref. [24].
The dewetting processes of solutions and suspensions are more involved than those of a pure liquid because they involve several interdependent dynamical processes: transport of solute or colloids, transport of the solvent and evaporation or condensation of the solvent. As a consequence, one must distinguish between 'normal' convective dewetting and evaporative dewetting. Experimental studies performed with volatile solutions or suspensions of polymers [8,9], macromolecules [10-12], and colloids a few nanometers in size (referred to as "nanoparticles") [5-7,25] describe a variety of richly structured deposits of the solutes. One may observe labyrinthine and polygonal network structures similar to the structures observed following "classical" dewetting. As the solvent evaporates, the solute remains dried onto the substrate and therefore "conserves" the transient dewetting pattern $[6,10]$. However, the solute is not just a passive tracer: it may influence the thresholds and the rates of the initial film rupture processes. Most importantly, it may also destabilize the straight dewetting fronts and trigger the creation of strongly ramified structures-a process observed in many different systems [7-9,26].

For instance, the dewetting of films of a suspension of thiol-coated gold nanoparticles in toluene may result in the deposition of the nanoparticles in branched finger patterns. The precise properties of these depend on the strength of the attraction between the colloidal particles [7]. Employing contrast-enhanced video microscopy to study the dynamics of the system, initially the receding of a mesoscopic dewetting front (equivalent to the receding three phase contact line) is observed, leaving behind an unstable ultrathin postcursor film, having a thickness similar to the diameter of the nanoparticles. Subsequently, the postcursor film breaks, forming a pattern of holes that themselves grow in an unstable manner, resulting in an array of branched structures. Note that the mesoscopic front may also be unstable-an effect that does not concern us here.

Theories for modeling such processes are rather limited at the present time. Hydrodynamical models of dewetting by thin films based on a long-wave approximation [24,27] provide a mesoscale description of the liquid film and can ac- 
count for evaporation of volatile liquids [28] and for the presence of a solute [29]. However, they are not able to describe the processes occurring in the postcursor film as they do not account for the interactions between the solute particles and between the solute particles and the solvent. Alternatively, to describe these processes one may employ two-dimensional (2D) kinetic Monte Carlo (KMC) lattice models that focus solely on the dynamics of the solute diffusion and the solvent evaporation [30-32]. The neglect of convective solvent transport can be justified based on estimates comparing its influence with the one of evaporative solvent transport [32]. However, so far the 2D KMC models have not incorporated diffusive solvent transport that might be important in the postcursor layer.

Here we present an alternative description for the structure formation in the postcursor film that does not have the limitations of the previous approaches. We develop a 2D dynamical density functional theory (DDFT) [33-36] to describe the coupled dynamics of the density fields of the liquid $\rho_{l}(\mathbf{r}, t)$ and the nanoparticles $\rho_{n}(\mathbf{r}, t)$. In this approach, diffusive liquid transport can be incorporated in a straightforward manner enabling us to go beyond previous 2D KMC studies and examine its influence. To construct the DDFT model, we (i) develop via coarse graining an approximation for the underlying free-energy functional of the system and (ii) form equations governing the dynamics of the two density fields. These are able to account for the nonconserved and conserved aspects of the dynamics, i.e., phase change and diffusive transport processes, respectively.

In order to compare results with the established KMC lattice model [30-32], we start from the lattice Hamiltonian to develop a mean-field (Bragg-Williams) approximation for the free-energy functional $[35,37]$. Expressing the interaction terms (sums over neighboring lattice sites) as gradient operators [35], the following semigrand [38] free-energy functional is obtained:

$$
\begin{aligned}
F\left[\rho_{l}, \rho_{n}\right]= & \int d \mathbf{r}\left[f\left(\rho_{l}, \rho_{n}\right)+\frac{\varepsilon_{l l}}{2}\left(\nabla \rho_{l}\right)^{2}+\frac{\varepsilon_{n n}}{2}\left(\nabla \rho_{n}\right)^{2}\right. \\
& \left.+\varepsilon_{n l}\left(\nabla \rho_{n}\right) \cdot\left(\nabla \rho_{l}\right)-\mu \rho_{l}\right]
\end{aligned}
$$

where $f\left(\rho_{l}, \rho_{n}\right)=k_{B} T\left[\rho_{l} \ln \rho_{l}+\left(1-\rho_{l}\right) \ln \left(1-\rho_{l}\right)\right]+k_{B} T\left[\rho_{n} \ln \rho_{n}\right.$ $\left.+\left(1-\rho_{n}\right) \ln \left(1-\rho_{n}\right)\right]-2 \varepsilon_{l l} \rho_{l}^{2}-2 \varepsilon_{n n} \rho_{n}^{2}-4 \varepsilon_{n l} \rho_{n} \rho_{l}$, includes entropic contributions and various interaction terms- the parameters $\varepsilon_{i j}$, where $i, j=n, l$, are the energies for having neighboring pairs of lattice sites occupied by species $i$ and $j$, respectively, $T$ is the temperature, $k_{B}$ is Boltzmann's constant, and we have set the lattice spacing $\sigma=1$. Note that $F$ in Eq. (1) can also be obtained by making a gradient expansion of the (nonlocal) free-energy functional of a continuous system [37]. The rate of evaporation of the liquid from the substrate is determined by the chemical potential $\mu$ in the reservoir (i.e., in the vapor above the substrate). When the temperature is sufficiently low and the chemical potential $\mu=\mu_{\text {coex }}$, we observe coexistence between a thick (high density) and a thin (low density) liquid film. In Fig. 1(a) we display the limit of linear stability (spinodal) and the equilibrium coexistence curve (binodal) for the pure liquid (i.e.,
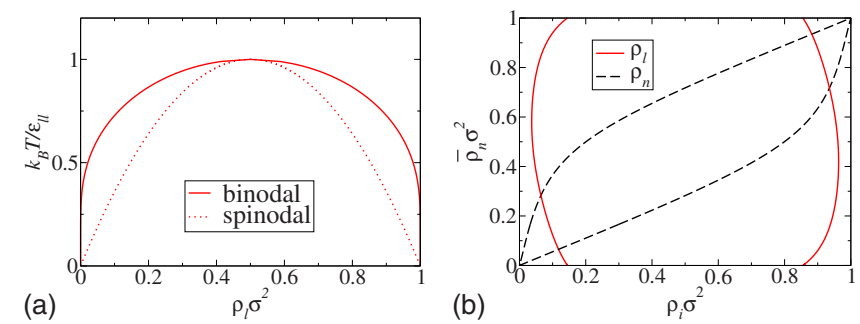

FIG. 1. (Color online) (a) Phase diagram of the pure liquid in the plane spanned by the density $\rho_{l}$ and temperature $T$. (b) Liquid and nanoparticle densities $\rho_{l}$ and $\rho_{n}$ at coexistence as a function of the average nanoparticle density $\bar{\rho}_{n}$ for $\varepsilon_{l l} / k_{B} T=1.25, \varepsilon_{n l} / k_{B} T=0.6$, and $\varepsilon_{n n}=0$.

with $\rho_{n}=0$ ). To indicate the influence of the solute we plot in Fig. 1(b) the densities $\rho_{l}$ and $\rho_{n}$ at coexistence for a fixed temperature $k_{B} T / \varepsilon_{l l}=0.8$ as a function of the average concentration $\bar{\rho}_{n}=\frac{1}{2}\left(\rho_{n}^{a}+\rho_{n}^{b}\right)$, where $\rho_{n}^{a}$ and $\rho_{n}^{b}$ are the densities of the nanoparticles in the coexisting $a$ and $b$ phases. For further details concerning the phase diagram and its topology see Ref. [39].

At equilibrium, the derivative $\mu_{n} \equiv \delta F\left[\rho_{n}, \rho_{l}\right] / \delta \rho_{n}$ is a constant, corresponding to the chemical potential of the nanoparticles. However, when the system is out of equilibrium, $\mu_{n}$ may vary along the substrate. We assume that the thermodynamic force $\nabla \mu_{n}$ drives the dynamics of the nanoparticles and that the nanoparticle current is $\mathbf{j}=-M_{n} \rho_{n} \nabla \mu_{n}$, where $M_{n}\left(\rho_{l}\right)$ is a mobility coefficient. This expression for the current, together with the continuity equation, yields the time evolution equation for the nanoparticle density profile:

$$
\frac{\partial \rho_{n}}{\partial t}=\nabla \cdot\left[M_{n} \rho_{n} \nabla \frac{\delta F\left[\rho_{n}, \rho_{l}\right]}{\delta \rho_{n}}\right] .
$$

This equation may also be obtained by assuming overdamped stochastic equations of motion for the nanoparticles $[33,34]$. To model the fact that nanoparticles do not diffuse over the dry substrate (when $\rho_{l}$ is small) we set the mobility $M_{n}\left(\rho_{l}\right)$ to switch at $\rho_{l}=0.5$ (smoothly) from zero for the dry substrate (low $\rho_{l}$ ) to $\alpha$ for the wet substrate (high $\rho_{l}$ ). Note that our results are not sensitive to the precise form of $M_{n}\left(\rho_{l}\right)$.

For the liquid, the density may change either by evaporation from or condensation to the substrate (nonconserved dynamics) or may diffuse over the substrate (conserved dynamics). The latter dynamics is treated in a manner analogous to that for the nanoparticles. For the nonconserved dynamics, we assume a standard form [40], i.e., that the change in the density over time is proportional to $-\left(\mu_{\text {surf }}\right.$ $-\mu)=-\delta F\left[\rho_{n}, \rho_{l}\right] / \delta \rho_{l}$, where $\mu_{\text {surf }}(\mathbf{r}, t)$ is the local chemical potential of the liquid on the substrate. Combining these two contributions, we obtain the time evolution equation of the liquid density profile:

$$
\frac{\partial \rho_{l}}{\partial t}=\nabla \cdot\left[M_{l}^{c} \rho_{l} \nabla \frac{\delta F\left[\rho_{n}, \rho_{l}\right]}{\delta \rho_{l}}\right]-M_{l}^{\mathrm{nc}} \frac{\delta F\left[\rho_{n}, \rho_{l}\right]}{\delta \rho_{l}} .
$$

We assume that the two mobility coefficients $M_{l}^{c}$ and $M_{l}^{\mathrm{nc}}$ are constants. In what follows we set $k_{B} T=1$ and $M_{l}^{\mathrm{nc}}=1$. Note 

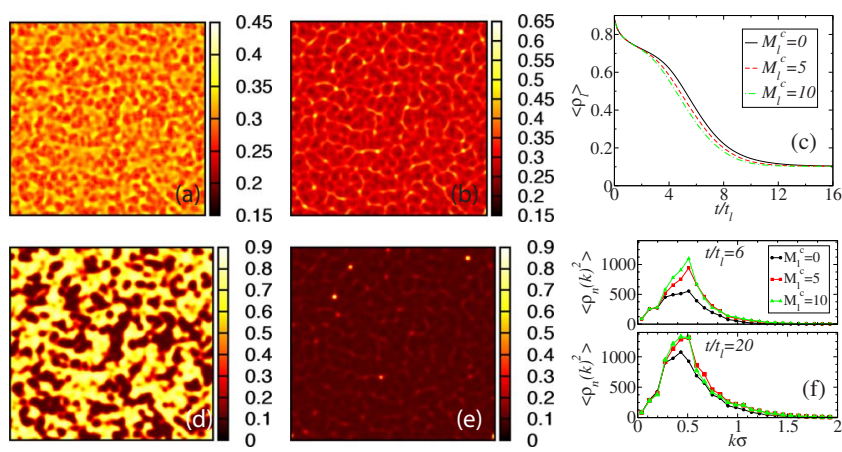

FIG. 2. (Color online) Results for spinodal evaporative dewetting of a nanoparticle suspension. (a) and (b) are typical nanoparticle density profiles, for times $t / t_{l}=6$ and 20 , and (d) and (e) are the corresponding liquid density profiles, for $M_{l}^{c}=0$. The domain size is $200 \sigma \times 200 \sigma$. (c) and (f) give the corresponding time evolution of the mean liquid density $\left\langle\rho_{l}\right\rangle$ and the structure factor $\left\langle\rho_{n}(k)^{2}\right\rangle$ for various values of $M_{l}^{c}$. The remaining parameters are $\varepsilon_{l l} / k_{B} T=1.25$, $\varepsilon_{n l} / k_{B} T=0.6, \varepsilon_{n n}=0, \alpha=0.4 M_{l}^{\mathrm{nc}} \sigma^{4}, \mu / k_{B} T=-3.4$, and $\left\langle\rho_{n}\right\rangle=0.3$.

that in the low density limit, when $\rho_{n} \rightarrow 0$ and $\rho_{l} \rightarrow 0$, the conserved part in both Eq. (2) and Eq. (3) corresponds to Fickian diffusion.

Before discussing results from our theory, we first make a couple of comments about the status of the theory. First, we note that Eq. (1) constitutes a simple "zeroth-order" meanfield approximation for the free energy of the system and omits (for example) terms such as $\ln \left(1-\rho_{n}-\rho_{l}\right)$ which describe the excluded area correlations between the liquid and the nanoparticles. Second, due to the fact that we derive the theory from the (already) coarse-grained lattice Hamiltonian rather than by integrating over degrees of freedom (coarse graining) in the full DDFT theory for the three-dimensional liquid film, the theory cannot be regarded as a "fully" microscopic theory. The reason that we have modeled the system using this simple theory is because we seek to address the basic question of what physics drives the behavior displayed in the experiments. In this work we choose to start from the lattice theory, in order to compare with the KMC. However, as future work we plan to go beyond the lattice theory. Our goal here is not to construct a model describing every detail of these systems; instead we seek to examine what physics is involved in determining the observed pattern formation. Nonetheless, this DDFT does allow us to investigate the time evolution of the postcursor film of an evaporating nanoparticle suspension under fewer restrictions than the $\mathrm{KMC}$ model. To discuss the importance of liquid transport within the layer we compare results obtained for different liquid mobilities $\left(M_{l}^{c}>0\right)$ and results without liquid transport (i.e., setting $M_{l}^{c}=0$ ). We focus on three examples: (i) spinodal dewetting (see Fig. 2), (ii) dewetting via nucleation of holes in an initially flat film (see Fig. 3), and (iii) the unstable receding of an evaporative dewetting front, which exhibits branched fingering (see Figs. 4 and 5).

Figure 2 shows snapshots from a purely evaporative spinodal dewetting process. Panel (c) gives the evolution of mean liquid density with time, $\left\langle\rho_{l}\right\rangle \equiv A^{-1} \int d \mathbf{r} \rho_{l}(\mathbf{r}, t)$, where $A$ is the area of the substrate, and panel (f) gives the nanoparticle structure factor $S(k) \equiv\left\langle\rho_{n}(k)^{2}\right\rangle$, where $\rho_{n}(k)$ is the Fou-
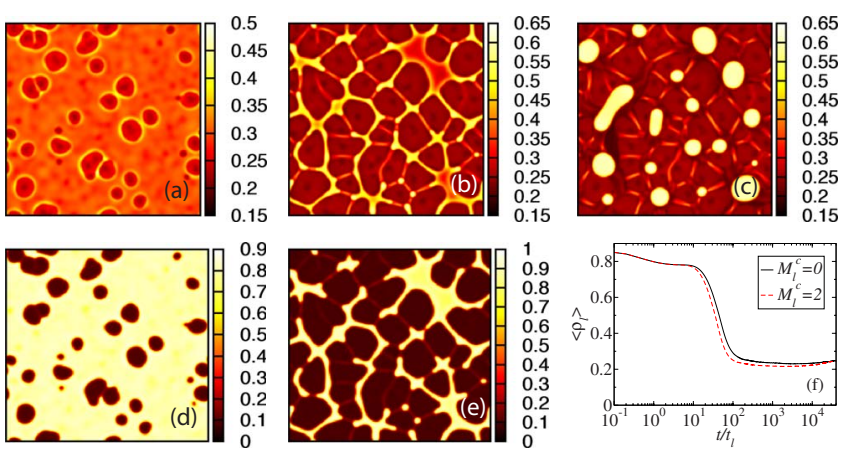

FIG. 3. (Color online) Density profiles for evaporative dewetting in the nucleation regime. (a)-(c) are the nanoparticle density profiles at times $t / t_{l}=20,80$, and $4000,(\mathrm{~d})$ and (e) are the liquid profiles for $t / t_{l}=20$ and 80 , for $M_{l}^{c}=2$ and $\mu / k_{B} T=-3.33$; remaining parameters are as in Fig. 2. In (f) we plot the average density of the liquid on the substrate $\left\langle\rho_{l}\right\rangle$, as a function of time for this case and the case $M_{l}^{c}=0$. The system was initialized with the (discretized) density profiles: $\rho_{l}(x, y, t=0)=0.9+0.05 \chi, \rho_{n}(x, y, t=0)$ $=0.3+0.27 \chi$, where $\chi$ is a random number uniformly distributed on the interval $[-1,1]$. It is due to this random noise that the holes are nucleated in some places and not in others.

rier transform of $\rho_{n}(\mathbf{r})$. For small times, the unstable film develops a typical spinodal labyrinthine pattern with a typical wavelength $2 \pi / k_{\max }$ (note that the symmetry breaking in the density profiles in all our calculations is due to the addition of a small amplitude random noise to the density profiles at time $t=0$ and no noise is added at later times). The nanoparticles concentrate where the remaining liquid is situated. However, they are "slow" in their reaction: when $\rho_{l}$ already takes values in the range $0.08-0.83$, the nanoparticle concentration has only deviated by about $25 \%$ from its mean value. The film thins rapidly forming many small holes. The competition for space results in a fine-meshed network of nanoparticle deposits with a much higher concentration of par-
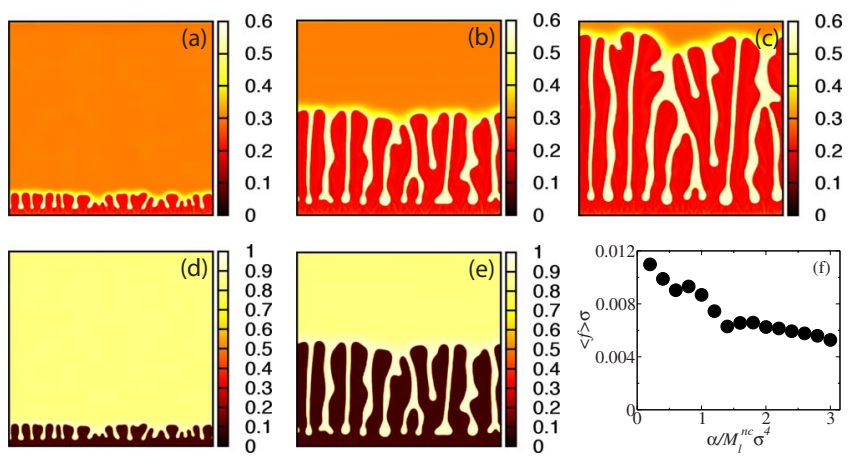

FIG. 4. (Color online) Density profiles from the evolution of an unstable dewetting front. (a)-(c) are the nanoparticle density profiles at times $t / t_{l}=2000,20000$, and 40000 , (d) and (e) are the liquid profiles for $t / t_{l}=2000$ and 20000 for $\alpha=0.2 M_{l}^{\mathrm{nc}} \sigma^{4}, \mu / k_{B} T$ $=-3.28$, and domain size $800 \sigma \times 800 \sigma$; remaining parameters are as in Fig. 2. The initial density profiles are the same as those in Fig. 3, except for $y<0$ we set $\rho_{l}=\rho_{n}=10^{-10}$, to create an initially straight dewetting front at $y=0$. In (f) we plot the mean finger number $\langle f\rangle$ as a function of the mobility coefficient for nanoparticle diffusion $\alpha$, for the case when $M_{l}^{c}=0$. 


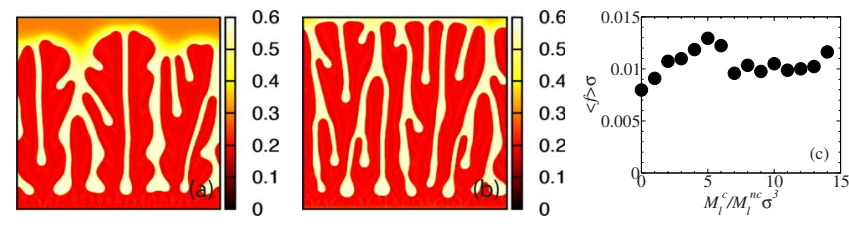

FIG. 5. (Color online) Nanoparticle density profiles from the evolution of an unstable dewetting front, for the time $t / t_{l}=20000$, (a) for $M_{l}^{c}=0$ and (b) $M_{l}^{c}=5$, for $\alpha=0.5 M_{l}^{\mathrm{nc}} \sigma^{4}, \mu / k_{B} T=-3.28$, and domain size is $800 \sigma \times 800 \sigma$; remaining parameters and initial density profiles are as in Fig. 4. In (c) we show the dependence of the mean finger number $\langle f\rangle$ on the mobility coefficient for liquid diffusion, $M_{l}^{c}$.

ticles at the network nodes-an effect that cannot be seen within the KMC model. Because the liquid wets the nanoparticles, some liquid always remains on the substrate. Accounting for solvent diffusion, the rate of the dewetting process is increased [see Fig. 2(c)] and leads to a more strongly modulated final pattern-i.e., the peaks in $S(k)$ are higher for $M_{l}^{c}>0$ than for $M_{l}^{c}=0$ [Fig. 2(f)].

Figure 3 shows snapshots from a dewetting process triggered by nucleation events. The holes nucleate at several arbitrary places due to the random initial noise in the density profiles and grow to form a random polygonal network of rims of highly concentrated solution. On a very long time scale the network coarsens into an array of drops. The influence of liquid transport can be seen in the final panel of Fig. 3 , where we display a plot of the average density of the liquid on the substrate as a function of (logarithmic) time. We see that the liquid is able to evaporate from the substrate faster and that for times $t / t_{l} \sim 10^{2}-10^{4}$, the total amount of liquid on the substrate is less when $M_{l}^{c}=2$ than when $M_{l}^{c}=0$. However, over very long times, the drops on the substrate slowly move and "eat-up" the network pattern. This process is faster with solvent diffusion, leading to a faster increase in $\left\langle\rho_{l}\right\rangle$ at long times. Since the liquid wets the nanoparticles, some liquid also recondenses back onto the substrate.

The final example in Figs. 4 and 5 is the evolution of the fingering instability for a receding dewetting front. The fingering instability is caused by a build up of the nanoparticles at the receding front, which collects the nanoparticles due to their attraction to the liquid. In Fig. 4(a) we see that at early times the initially straight front shows a rather short-wave instability; about 20 fingers can be seen. However, the finger pattern coarsens rapidly to a stationary pattern containing only about half the initial number of fingers. Intriguingly, the mean finger number remains constant although at the moving contact line new branches are created and old branches merge continuously. The occurrence of this phenomenon in the present continuum model (DDFT) is similar to results of the KMC [32] and proves that jamming of discrete particles is not a necessary mechanism for causing the instability. In Fig. 4(f) we show how the average number of fingers per unit length, $\langle f\rangle$, varies as a function of $\alpha$, the mobility coefficient of the nanoparticles on the wet substrate for the case when $M_{l}^{c}=0$. We see that as $\alpha$ is decreased, the number of fingers increases. This increase in $\langle f\rangle$ occurs because when the mobility of the nanoparticles is decreased the front "collects" more particles (less of them diffuse further from the front). The resulting region of high concentration solution at the front may be "dynamically unstable:" as the front velocity depends nonlinearly on the amount of particles collected, any fluctuation along the front may trigger a transverse instability.

Figure 5 shows that the finger number $\langle f\rangle$ depends nonmonotonically on the mobility coefficient for liquid diffusion, $M_{l}^{c}$. Although the overall trend is an increase in $\langle f\rangle$ with increasing $M_{l}^{c}$, there exists an intermediate region $\left(5 \lesssim M_{l}^{c} \lesssim 7\right)$ where $\langle f\rangle$ slightly decreases. The overall trend results from an increase in front velocity (due to the increase in $M_{l}^{c}$ ) at fixed particle diffusivity. However, we currently have no explanation for the intermediate slight decrease.

Note also that in all cases the instability may be strongly amplified when the particle interactions favor the clustering of the nanoparticles (this is not the case for the parameters used here), where the higher concentration at the receding front leads to a local demixing of nanoparticles and liquid that itself enforces the deposition of a highly branched finger pattern. In this "demixing regime" the instability is determined by the dynamics and the energetics of the system whereas for the case studied in Fig. 4 it mainly depends on the dynamics.

We note finally that the fingering process can be seen as a self-optimization of the front motion so that the average front velocity is kept constant by expelling particles into the fingers. A similar effect exists for dewetting polymer films [22], where surplus liquid is expelled from the growing moving rim which collects the dewetted polymer. However, front instabilities found for dewetting polymers only result in fingers without side branches [41] or fields of droplets left behind [22].

In this paper we have developed a versatile DDFT that is capable of describing the pattern formation observed in evaporating dewetting thin films of suspensions. Since our DDFT takes account of all the basic solvent and solute transport and phase change processes in a consistent manner, we believe that it will be the basis for many successful future studies of the behavior of suspensions and solutions at interfaces.

A.J.A. and M.J.R. gratefully acknowledge financial support by RCUK and EPSRC-GB, respectively. We acknowledge support by the EU via Grant No. PITN-GA-2008214919 (MULTIFLOW). 
[1] A. Giacometti, A. Maritan, and J. R. Banavar, Phys. Rev. Lett. 75, 577 (1995).

[2] J. Veysey and N. Goldenfeld, Nat. Phys. 4, 310 (2008).

[3] R. D. Deegan, Phys. Rev. E 61, 475 (2000).

[4] H. Malchow, S. V. Petrovskii, and E. Venturino, Spatiotemporal Patterns in Ecology and Epidemiology: Theory, Models, and Simulations (Chapman \& Hall/CRC, London, 2008).

[5] G. L. Ge and L. Brus, J. Phys. Chem. B 104, 9573 (2000).

[6] P. Moriarty, M. D. R. Taylor, and M. Brust, Phys. Rev. Lett. 89, 248303 (2002).

[7] E. Pauliac-Vaujour et al., Phys. Rev. Lett. 100, 176102 (2008).

[8] X. Gu, D. Raghavan, J. F. Douglas, and A. Karim, J. Polym. Sci., Part B: Polym. Phys. 40, 2825 (2002).

[9] G. Liu, C. F. Zhang, J. Zhao, and Y. X. Zhu, Langmuir 24, 7923 (2008).

[10] U. Thiele, M. Mertig, and W. Pompe, Phys. Rev. Lett. 80, 2869 (1998).

[11] I. I. Smalyukh, O. V. Zribi, J. C. Butler, O. D. Lavrentovich, and G. C. L. Wong, Phys. Rev. Lett. 96, 177801 (2006).

[12] L. Zhang, S. Maheshwari, H. C. Chang, and Y. X. Zhu, Langmuir 24, 3911 (2008).

[13] G. Reiter, Phys. Rev. Lett. 68, 75 (1992).

[14] R. Seemann, S. Herminghaus, and K. Jacobs, Phys. Rev. Lett. 86, 5534 (2001).

[15] M. Elbaum and S. G. Lipson, Phys. Rev. Lett. 72, 3562 (1994).

[16] Y. Gotkis, I. Ivanov, N. Murisic, and L. Kondic, Phys. Rev. Lett. 97, 186101 (2006).

[17] D. Quemada and C. Berli, Adv. Colloid Interface Sci. 98, 51 (2002).

[18] U. Thiele, M. G. Velarde, and K. Neuffer, Phys. Rev. Lett. 87, 016104 (2001).

[19] J. Becker et al., Nature Mater. 2, 59 (2003).

[20] C. Redon, F. Brochard-Wyart, and F. Rondelez, Phys. Rev. Lett. 66, 715 (1991).

[21] A. Sharma and G. Reiter, J. Colloid Interface Sci. 178, 383 (1996).

[22] G. Reiter and A. Sharma, Phys. Rev. Lett. 87, 166103 (2001).
[23] A. Münch and B. Wagner, Physica D 209, 178 (2005).

[24] Thin Films of Soft Matter, edited by S. Kalliadasis and U. Thiele (Springer, Wien, 2007).

[25] M. Maillard, L. Motte, A. T. Ngo, and M. P. Pileni, J. Phys. Chem. B 104, 11871 (2000).

[26] U. Thiele, Ph.D. thesis, Technische Universität Dresden, 1998.

[27] A. Oron, S. H. Davis, and S. G. Bankoff, Rev. Mod. Phys. 69, 931 (1997)

[28] A. V. Lyushnin, A. A. Golovin, and L. M. Pismen, Phys. Rev. E 65, 021602 (2002).

[29] M. R. E. Warner, R. V. Craster, and O. K. Matar, J. Colloid Interface Sci. 267, 92 (2003).

[30] E. Rabani, D. R. Reichman, P. L. Geissler, and L. E. Brus, Nature (London) 426, 271 (2003).

[31] C. P. Martin et al., Phys. Rev. Lett. 99, 116103 (2007).

[32] I. Vancea et al., Phys. Rev. E 78, 041601 (2008).

[33] U. Marconi and P. Tarazona, J. Chem. Phys. 110, 8032 (1999).

[34] A. J. Archer and M. Rauscher, J. Phys. A 37, 9325 (2004).

[35] J. F. Gouyet, M. Plapp, W. Dieterich, and P. Maass, Adv. Phys. 52, 523 (2003).

[36] Note that our DDFT shares some features in common with the phase field theories used to study solidification and other such processes—see, e.g., A. A. Wheeler, W. J. Boettinger, and G. B. McFadden, Phys. Rev. A 45, 7424 (1992).

[37] P. M. Chaikin and T. C. Lubensky, Principles of Condensed Matter Physics (Cambridge University Press, Cambridge, 1997).

[38] We denote it a "semigrand" free energy because the free energy is developed in an ensemble where the liquid is treated grand canonically (it is coupled to a reservoir with chemical potential $\mu$ ), whereas the fixed number of nanoparticles are treated canonically.

[39] D. Woywod and M. Schoen, Phys. Rev. E 73, 011201 (2006).

[40] J. S. Langer, in Solids Far from Equilibrium, edited by C. Godreche (Cambridge University Press, Cambridge, 1992), pp. 297-363.

[41] G. Reiter, Langmuir 9, 1344 (1993). 